\title{
A NON-EXCEPTIONAL ELEMENT OF WIENER SPACE
}

W. S. LOUD

Let $C$ denote the space of functions $x(t)$, continuous on $0 \leqq t \leqq 1$ with $x(0)=0$. For each element of $C$ let

$$
\sigma_{n}(x)=\sum_{j=1}^{2 n}\left[x\left(\frac{j}{2^{n}}\right)-x\left(\frac{j-1}{2^{n}}\right)\right]^{2} .
$$

Cameron and Martin [1] ${ }^{1}$ have shown that for almost all elements of $C$, in the sense of Wiener measure, the limit

$$
\int_{0}^{1}|d x(t)|^{2}=\lim _{n \rightarrow \infty} \sigma_{n}(x)
$$

exists and has the value $1 / 2$.

The purpose of this note is to construct an explicit example of an element of $C$ for which the above limit is equal to $1 / 2$. The example is of interest, since for most "ordinary" functions the value of the limit is zero. If $x(t)$ is of bounded variation on $0 \leqq t \leqq 1$, the limit is zero. If $x(t)$ satisfies for $0 \leqq t \leqq 1$ a uniform Lipschitz condition of order $\alpha$, where $\alpha$ is greater than $1 / 2$, the limit is zero, whereas it is possible for such a function to be everywhere nondifferentiable [2].

The present construction is a modification of one given by the author [2]. In the notation of [2] we use $A=\alpha=1 / 2$. Specifically let $g(t, h)$ be periodic of period $2 h$, equal to zero for even multiples of $h$, equal to one for odd multiples of $h$, and linear between. Let $x_{0}(t)$ be defined by

$$
x_{0}(t)=\frac{1}{2} \sum_{m=1}^{\infty} 2^{-m / 2} g\left(t, 2^{-m}\right) .
$$

$x_{0}(0)=0$, and $x_{0}(t)$ is continuous on $0 \leqq t \leqq 1$ by uniform convergence of the series. Thus $x_{0}(t) \in C$. We now show that

$$
\int_{0}^{1}\left|d x_{0}(t)\right|^{2}=\frac{1}{2}
$$

If the interval $0 \leqq t \leqq 1$ is divided into $2^{n}$ equal parts, the increments of $x_{0}(t)$ are the $2^{n}$ different values that can be obtained from all possible combinations of signs in the expression

Presented to the Society, May 1, 1954; received by the editors March 29, 1954.

1 The author has been informed that this result was found originally by P. Lévy. 


$$
\frac{1}{2} 2^{-n}\left( \pm 2^{n / 2} \pm 2^{(n-1) / 2} \pm \cdots \pm 2^{1 / 2}\right)
$$

This follows from the fact that only the first $n$ terms of the series for $x_{0}(t)$ will have increments different from zero, while the directions of the increments of the first $n$ terms will occur in all possible combinations. Now when we compute the sum of the squares of these $2^{n}$ increments, the cross-product terms occur as often positive as negative, so that only the squares of the terms contribute, and the sum of the squares of the increments is

$$
\begin{aligned}
\sigma_{n}\left(x_{0}\right) & =\frac{1}{4} 2^{-2 n} 2^{n}\left(2^{n}+2^{n-1}+\cdots+2\right) \\
& =\frac{1}{2}-\frac{1}{2^{n+1}} .
\end{aligned}
$$

Therefore

$$
\lim _{n \rightarrow \infty} \sigma_{n}\left(x_{0}\right)=\int_{0}^{1}\left|d x_{0}(t)\right|^{2}=\frac{1}{2}
$$

\section{BIBLIOGRAPHY}

1. R. H. Cameron and W. T. Martin, The behavior of measure and measurability under change of scale in Wiener space, Bull. Amer. Math. Soc. vol. 53 (1947) pp. 130137.

2. W. S. Loud, Functions with prescribed Lipschitz condition, Proc. Amer. Math. Soc. vol. 2 (1951) pp. 358-360.

UNIVERSity OF MinNESOTA 